\title{
License Plate Automatic Recognition System Based on MATLAB-GUI
}

\author{
Wei Xie ${ }^{1, *}$ and Yuwang $\mathrm{Wu}^{2}$ \\ ${ }^{I}$ College of Science, Guilin University of Technology, Guilin, Guangxi, 541004, P.R. China \\ ${ }^{2}$ Department of Mathematics and Econometrics, Hunan University of Humanities Science and Technology, Loudi, Hu- \\ nan, 417000, P.R. China
}

\begin{abstract}
With the rapid development of the transportation industry in our country, traffic management in China has gradually transformed into a digital and intelligent system. In traffic management digitization, intelligence is one of the most important links of license plate automated recognition. Considering this problem, a license plate automatic recognition algorithm based on the image processing technology is established, and then a corresponding GUI system is developed using MATLAB software system. Experimental results show that the system can be used more quickly and accurately for license plate recognition.
\end{abstract}

Keywords: Image processing, license plate automatic recognition, MATLAB.

\section{INTRODUCTION}

With the increasing number of vehicles various traffic problems on the current road management system are also increasing. One of the most serious issue is the traffic management. At present, to improve the efficiency of traffic department management and to ease the traffic pressure is the scholar's research direction. But in this article, it is of most importance to develop a set of prefect and intelligent traffic management system. At the same time, our country's computer technology and the level of automation information processing ability is also continuously improving which provides a good technical support for the development of intelligent traffic management system. In this kind of background, intelligent transportation system has become the main trend of the development of China's road traffic management.

The research of automatic recognition of vehicle license plate has developed earlier internationally. Since the 80 's, many scholars did the research on the license plate recognition by using the method of image processing $[1,2]$, but the final recognition results always are far from the ideal effect because the automatic license plate recognition system is not complete. Though there are many domestic types of license plates which contain Chinese characters, it's not unified and sometimes composed of numbers entirely. There are many international researches from which we can learn several components of the vehicle license plate recognition system and the method of template matching used in Character recognition, etc. In China, many scholars have done research on The license plate recognition algorithm and system based on Image processing technology and have abundant research results [3-10].
Although the research achievements are numerous, most of the research is limited in theory, there are few which have practical programming. In the programming of the results, the recognition speed and the precision of the algorithm are yet to be improved, and License plates recognition is not ideal in a complex shot environment. Hence, this paper establishes a more practical and fast license plate recognition algorithm, and then uses MATLAB software to design and develop a simple and rapid automatic license plate recognition system.

\section{AUTOMATIC LICENSE PLATE RECOGNITION ALGORITHM BASED ON IMAGE PROCESSING}

License plate automatic recognition system mainly includes image acquisition, image preprocessing, license plate locating, character segmentation, character recognition and output results etc. The process is shown in Fig. (1).

Each step of the above six steps are essential for the whole license plate automatic recognition system and each of these steps are linked together. Therefore, to complete the whole system's identification function, a good job needs to be done in every step. Image processing and recognition of the characters are the key steps for the recognition rate and recognition speed in the whole system. In many automatic license plate recognition products, it is very difficult for the recognition speed and recognition rate to improve at the same time. Because Image processing technology is insufficient and character recognition algorithm is not precise enough, especially the Chinese character recognition. Therefore, the main task of this topic is using the powerful image processing technology of MATLAB to improve the recognition speed and establish a fast and accurate character recognition algorithm. 


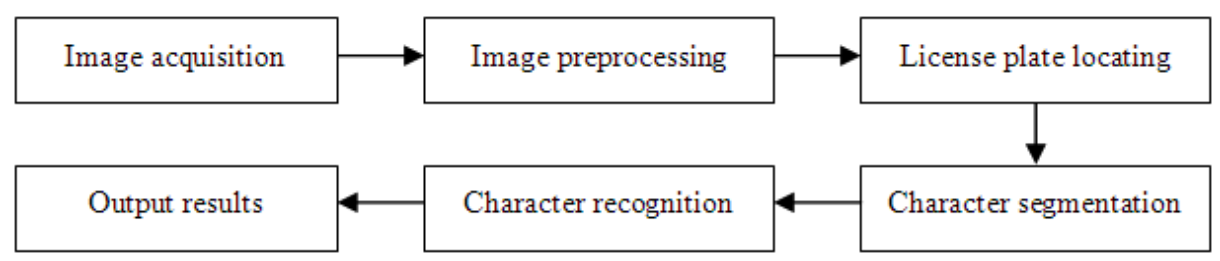

Fig. (1). The work flow chart of automatic license plate recognition system.

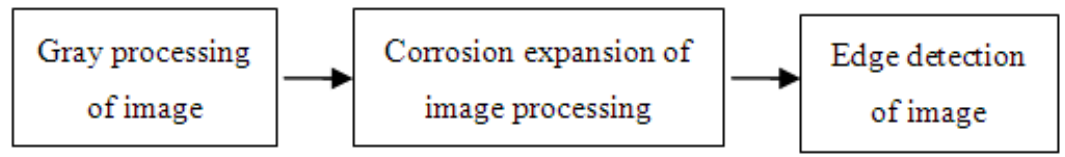

Fig. (2). The algorithm flow chart of Image preprocessing.

\subsection{The Image Acquisition}

This step is usually performed under the certain trigger conditions image acquisition equipment acquires an image and uses the computer network to transmit to the vehicle license plate automatic recognition system. But due to the constraints in this topic, we don't have these hardware devices, so we use the high-definition cameras to simulate this process. The most common image formats currently used are BMP, JPG and so on. JPG is currently the most widely used image and transmission format, and most camera images are saved as JPG format, this topic collected images in JPG format.

\subsection{The Algorithm of Image Preprocessing}

In order to reduce the amount of calculation in the process of image processing, we need to do image gray processing. Firstly, using image corrosion technology to remove the effect of the image edge burr and saw tooth. Secondly, filling the pit and the missing parts of the image by using the method of image expansion because on the condition of the uneven illumination and dust influence of optical path, the automotive original image that camera collected will be blurred, edge serrated, or pictured with potholes, etc. Finally, we also need to deal with the noise of the original image for the external environment noise and the noise of the electronic device will interfere with the pictures. The color gradient changes, obviously, because the license plate is usually blue or yellow, but the Identification of brightness changes of the digital image using edge detection. The algorithm flow chart is shown in Fig. (2).

\subsection{The Algorithm of License Plate Locating}

After the image preprocessing of the vehicle image, most of the region is still a background image, without any sense of recognition, in order to save time and memory space of the system identification, we should extract what we need from the original image and discard what we don't need. After the original image dealing with the gray processing and edge detection, the area of license plate are already clear. We use mathematical morphology and color positioning to do image processing and pattern recognition, the method of Mathematical morphology uses the elements with a certain shape to measure and extract the corresponding shape in the image for analysis and recognition, and noise reduction., Keeping the information of the original image and extracting image skeleton which have edge smoothing.

This step is mainly to calculate the car image after preprocessing. Firstly, we use the method of statistical color pixel to determine the range of gray scale of blue license plate that corresponds to RGB, next the number of Pixel in the rows and columns are counted within the limitation of the color to determine the region of license plate. Secondly, we get orientation matrix of the license plate region, and then remove the unnecessary part removal to obtain the license plate images that are located already. The main technology is to calculate the projection area of the edge image to find the peak point and approximately determine the position of the license plate, then calculate aspect ratio of the connected domain and weed out the connected domain that doesn't fit in the threshold value range for obtaining the region of license plate. The algorithm flow chart is shown in Fig. (3).

\subsection{The Algorithm of Character Segmentation}

The system can't identify the car license plate image directly after separated, we also need to make each of the char-

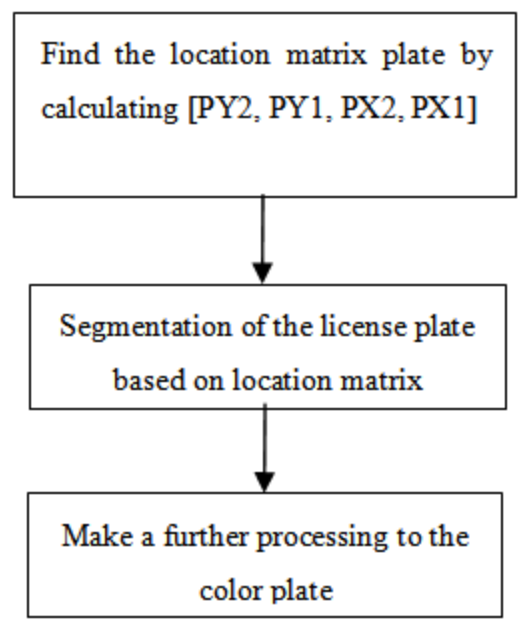

Fig. (3). The flow chart of license plate localization algorithm. 


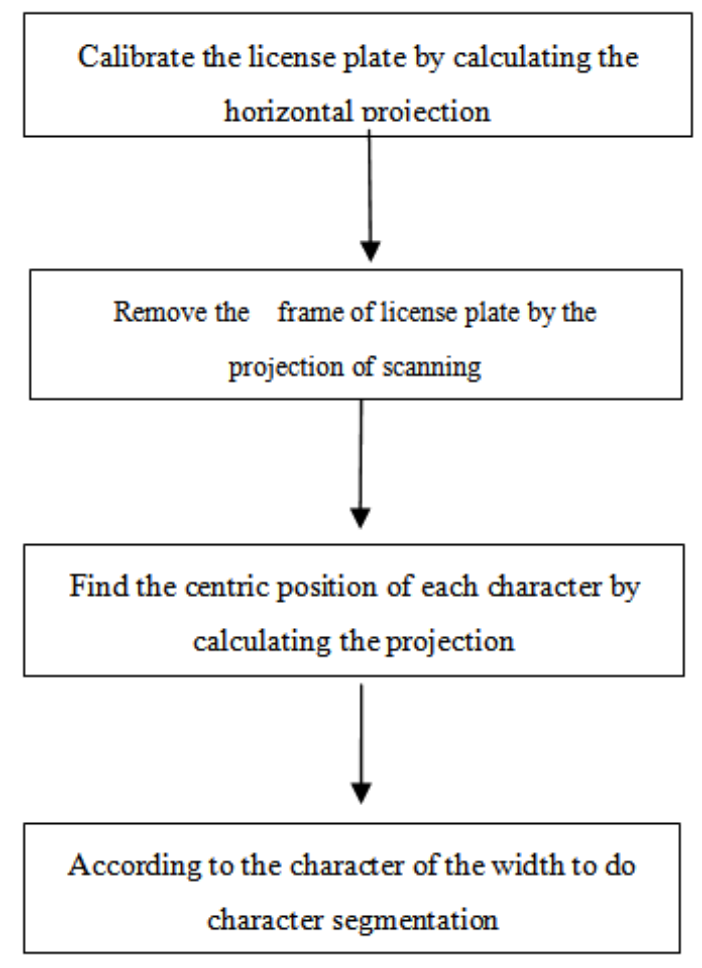

Fig. (4). The flow chart of character segmentation algorithm.

acters on the license plate segmentation independently and completely to gain a single character image and then the system recognizes each character separately. Character segmentation has an important role in the automatic license plate recognition system. It conducts character segmentation on the basis of license plate localization and uses segmentation results for character recognition.

Firstly, we make the image linearization, then carry it to vertical projection, because in projection drawing, there are obvious waveform changes which are similar to the peak and valley, determine the position of each character according to the characteristics of the valley and peak by scanning wave shape from left to right on the projection drawing. Finally, we calculate the vertical peak and detect the rational character's aspect ratio. The algorithm flow chart is shown in Fig. (4).

\subsection{The Algorithm of Character Recognition}

Presently the license plate character recognition algorithm is mainly a recognition algorithm based on template matching and neural network. The main steps of recognition algorithm based on template matching are: First of all, we make the characters that remain to be identified as linear, and then can scale down the template character pixel size as database template, next, we match all of the templates, finally we choose the best matching, as a result. The main characteristic is to realize the simple template matching. When the license plate characters clear and concise, even with some defects such as stains interference, the recognition rate is quite high. Although the neural network algorithm for digital part have a high degree of recognition, but are too compli- cated. Therefore, we select the template matching method as the main method of license plate character recognition.

MATLAB powerful data processing ability makes this process simple and quick when using the method of template matching to identify characters. After completing a series of processing images with MATLAB, we can obtain the character images which remain for recognition. It will extract characteristic values from the matrix Card (I, J) of character images, then we compare with the corresponding characteristic quantities of the template matrix loaded T (I, J), calculate normalized relations with each other. The major mutual relationship has the highest similarity degree between them and the image can be attributed to the corresponding class. Of course, it also can calculate the distance between the image and the template feature, using the minimum distance determines the class they belong to. Here, we calculate the similarity between character and template by using the method of template character with the unknown character subtraction. First, we take out the whole character templates, and then we take out the unknown characters one by one and with the template character subtraction, add up the number of 0's. The more the number of 0's the more the matching, last, we save the higher matching degree's name of the template as the recognition result.

We divide the license plate character into seven parts. The first character of the license plate is a Chinese character which represents the province that vehicle belongs to. They are letters or numbers besides the characters. There are more than 40 Chinese characters signifying province, military etc, 24 Capital English letters except I and $\mathrm{O}$ and numbers. In order to make it experimentally convenient, we establish 5 provinces, 24 letters and 10 numbers template when constructing character template library. We get other template design methods in the same way. The algorithm flow chart is shown in Fig. (5).

\section{DESIGN OF LICENSE PLATE RECOGNITION SYSTEM BASED ON MATLAB-GUI}

\subsection{The Main Interface of System}

In this system, the left window is used for displaying original image, in order to contrast the effect in the recognition process; the right window is used for displaying the whole recognition process. There are two buttons in the lower left quarter, one is used to open the car picture required to identify and the other is used to start recognition. There are two buttons in the lower right quarter, one is used to pause program, the other is used to exit the program. With this part, some important process playback of license plate recognition can be made flexible. The part of the program (see Annex specific code) cannot be used in the process of Program operation, only when the whole recognition process is finished, it can be used.

For specific operations: click the Open button to open the picture dialog box. Choose a picture you need to identify the license plate of the car with, then point Start button to start the recognition. You can click Pause to pause the recognition 


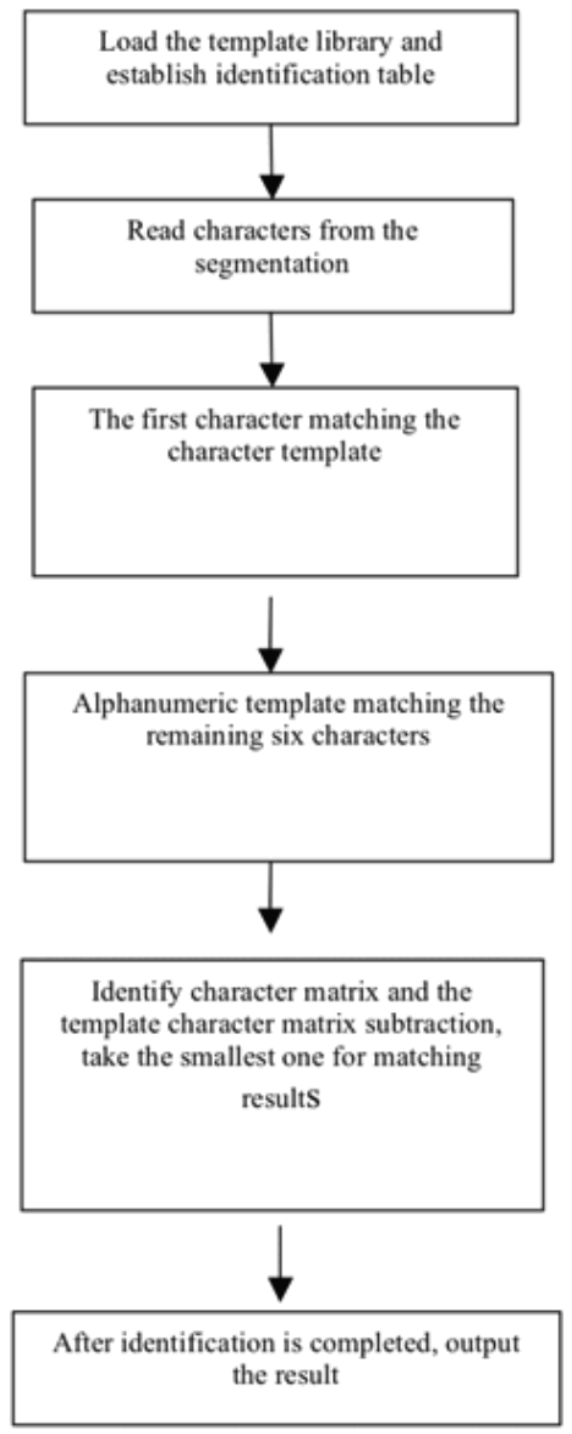

Fig. (5). The flow chart of character recognition algorithm.

process in the identification process, but you cannot click Pause again to continue the program execution, we can only click the Start restart recognition. This is controlled by MATLAB single threaded programming's limitation, the user experience is deficient. Of course, we can also exit at point Exit program. Main interface diagram of the System is shown in Fig. (6).

\subsection{The Interface of Image Preprocessing}

The interceptions of the screen are the two most important pictures in pretreatment. Other processes are completed by the function of MATLAB, which are relatively simple; therefore, they are not displayed in this article. Image is left simple black and white after the grayscale, this reduces many factors which influence image recognition, the effect is shown in Fig. (7).

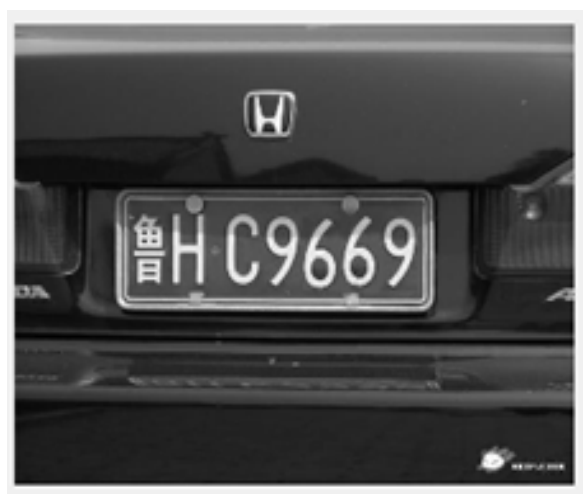

(a) The grayscale process

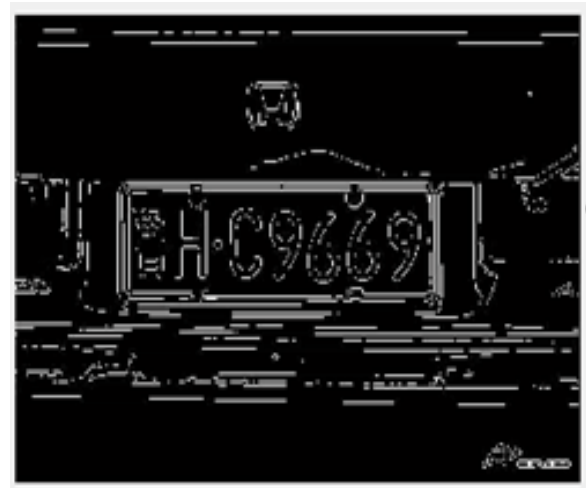

(b) Edge detection of canny

Fig. (7). Pretreatment of the image.

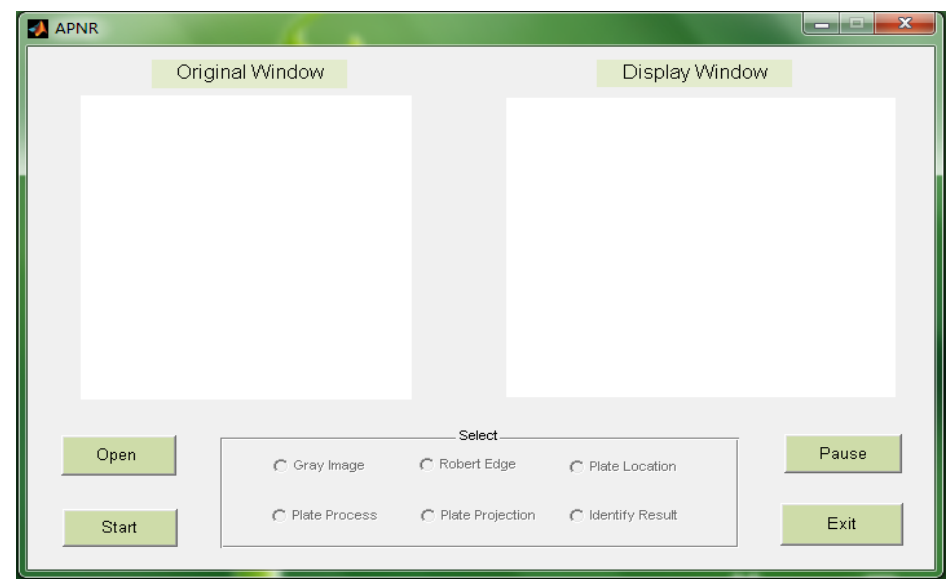

Fig. (6). The main interface diagram of the system. 


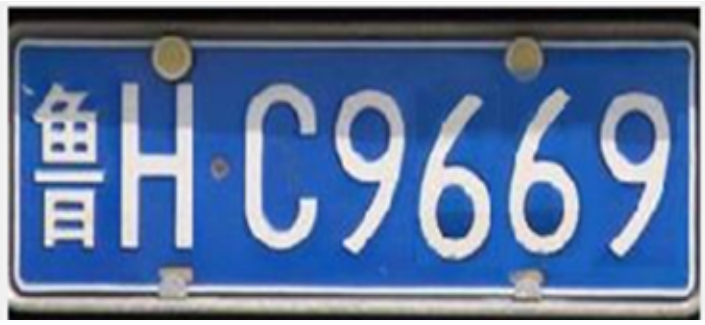

Fig. (8). License plate location.

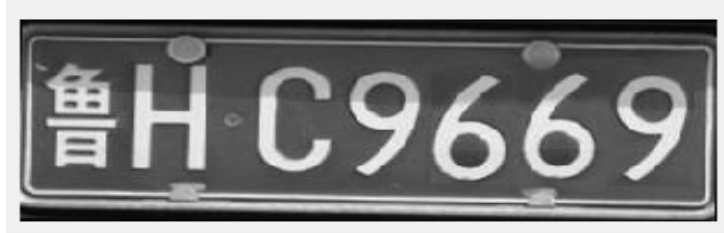

Fig. (9). License plate optimization.

\section{鲁H C9669|}

Fig. (10). The vertical projection of the license plate.

\subsection{The Interface of License Plate Location and Calibra- tion}

In this experiment, we select the samples of high quality which have no stains and damage and the angles of the photos are also good. So the car license is extremely accurate after localization. If the license plate has stains or defects the program can also locate it as correct as long as it won't affect the character recognition degree. When the shooting angle of the camera in the picture has deviation, the program can be effective, because in the algorithm of vehicle license plate location, threshold of the license plate's horizontal $\mathrm{X}$ and vertical $Y$ has fault tolerance. Effect of the license plate positioning is shown in Fig. (8).
In this part, we make further processing to the separated license plate in order to calibrate angle problems which happen during the filming process. This step makes the license plate do angle rotation. To remove the factors which have nothing to do with the license plate it carries the gray processing on the license plate. So, the influence factors of character segmentation can be a minimum. The license plate optimization process is shown in Fig. (9).

Projection is the most important part in character segmentation because, accurate or not, it is directly related to whether the license plate can be separated into seven characters. Through the projection, it can deal with the license plate frame, rivets and other interference factors. Furthermore, it reduces the inclination of the license plate caused by the angle of shooting. Plate vertical projection is shown in Fig. (10).

\subsection{The Interface of License Plate Recognition}

This part shows the character segmentation and recognition of vehicle license plate. We can see from the right side window of Fig. (11), the license plate is cut into seven clearly visible parts only containing characters. The whole process includes cutting the matrix character by calculating the blank area between the license plate characters firstly, then matching with the template library and identification before it is normalized, finally saving the recognition results. The recognition process is shown in Fig. (11).

The final recognition results as shown in Fig. (12).

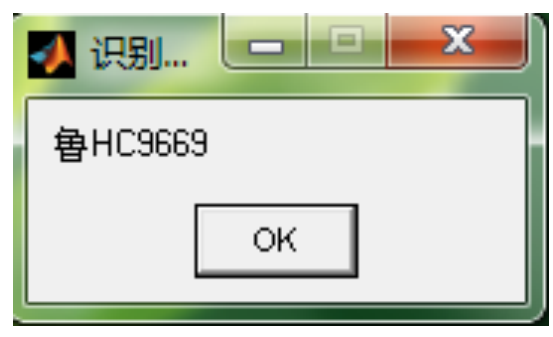

Fig. (12). The final recognition results.

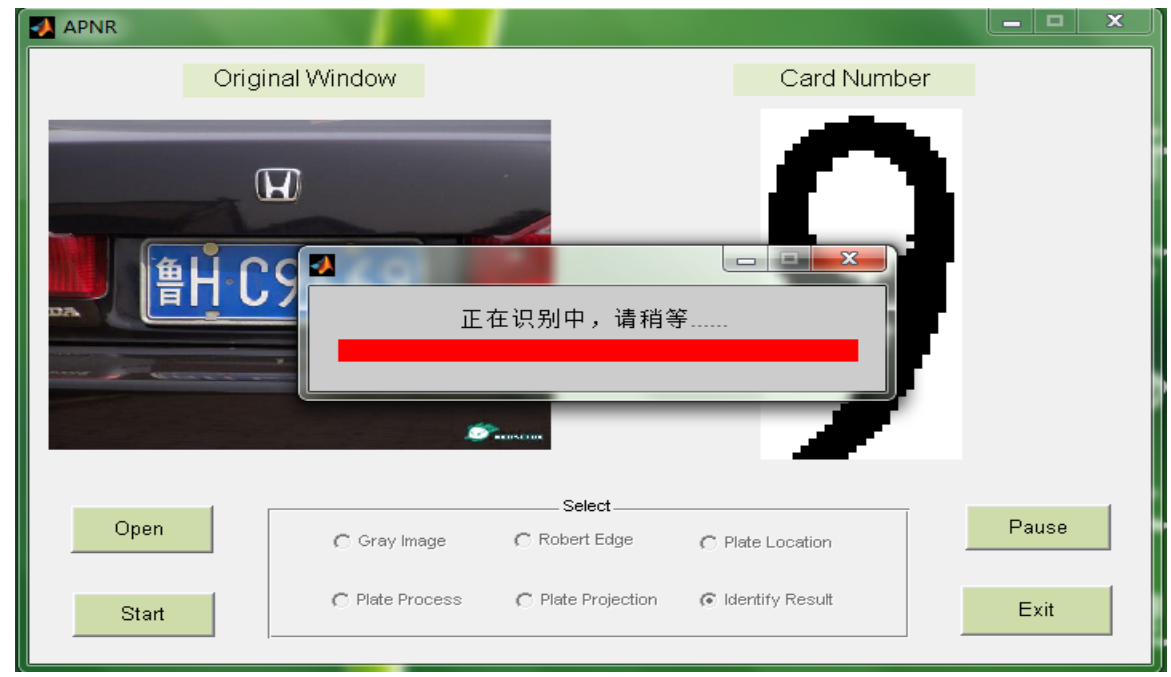

Fig. (11). The process of license plate recognition. 


\section{CONCLUSION}

Based on the problems of license plate recognition technology, the recognition of license plate is not accurate enough, character recognition accuracy is not high and the overall recognition rate is not ideal etc. This paper first performs grayscale processing to the image, and then does the pretreatment of image edge detection by using the high accuracy and high speed method of canny operator. Then through calculation of edge image's projection area, finding valley and peak and locating the license plate, we accurate, through the determination of license plate location matrix and by the method of statistical color pixel matrix, finish the location of license plate and scan and calculate the projection matrix of the license plate. We calculate the center position and width between the characters after the frame removal of the license plate. We get the position of the matrix of each single character by cutting the license plate and completing the character segmentation. Finally, we read the segmented characters to the system and load it into the recognition template library of pre-established characters, match each of them after normalization and save the maximum matching degree of the character as the recognition results.

Of course, this system has much room for improvement, not only can it use the programming skills of the global variables of MATLAB to simplify the variables, it can also use extensive matrix operation instead of multiple cyclic to reduce algorithm. It can also do statistical analysis of threshold in different parts of license plate location, such as count the threshold when positioning and get its optimal range, all of these can improve the efficiency and accuracy of license plate recognition.

\section{ACKNOWLEDGEMENTS}

Project Supported by Program to Sponsor Teams for Innovation in the Construction of Talent Highlands in Guangxi Institutions of Higher Learning ([2011]47).

\section{REFERENCES}

[1] S. Johnson, and B.M. Bird, "Number-plate matching for automatic vehicle identification", In: IEE Colloquium on Electronic Images and Image Processing in Security and Forensic Science, 1990, p. 4.

[2] R.A. Lotufo, A.D. Morgan, and A.S. Johnson, "Automatic numberplate recognition", In: IEE Colloquium on Image Analysis for Transport Applications, 1990. p. 6.

[3] J. Rong, W.J. Wang, and J.H. Chen, "Design and implement of the intelligent license plate recognition system", Microcomp. Its Appl., vol. 1 , pp. 50-51, 2005.

[4] X.C. Luo, G.X. Liu, Y.Q. Feng, and B.H. Shen, "A vehicle license plate recognition method based on the characteristics of vehicle license plate", J. South China Univ. Tech. (Nat. Sci.), vol. 31, pp. 7073, 2003.

[5] L.H. Xu, Y.P. Chen, and K.D. Xiu, "Static license plate recognition technology based on digital image processing", J. Jiangxi Univ. Sci. Tech., vol. 32, pp. 47-50, 2011.

[6] L. Wang, H.L. Wang, and L.H. He, "License plate recognition based on double-edge detection", Comp. Eng. Appl., vol. 49, pp. 169-173, 2013.

[7] Q. Qiu, "A license plate recognition system based on MATLAB image processing”, Mech. Eng., vol. 8, pp. 87-89, 2008.

[8] D. Zhao, J.H. Ding, "Recognition of car license plate based on MATLAB”, J. Dalian Polytech. Univ., vol. 28, pp. 303-304, 2009.

[9] X.P. L.U, and A.L. Chen, "Car license plate recognition Based on MATLAB”, Bull. Survey. Mapp., vol. 10, pp. 35-38, 2007.

[10] Y.H. Peng, G.T. Ding, and Q. Liang, "Multi-frame images integrated technology applied to license plate recognition", Microcomp. Inform., vol. 26, pp. 167-168, 2010.

\section{CONFLICT OF INTEREST}

The authors confirm that this article content has no conflict of interest.

Received: September 07, 2014

Revised: October 19, 2014

Accepted: November 20, 2014

(C) Xie and $\mathrm{Wu}$; Licensee Bentham Open.

This is an open access article licensed under the terms of the Creative Commons Attribution Non-Commercial License (http://creativecommons.org/licenses/by$\mathrm{nc} / 3.0 /$ ) which permits unrestricted, non-commercial use, distribution and reproduction in any medium, provided the work is properly cited. 